\title{
Vanillin rich fraction regulates LDLR and HMGCR gene expression in HepG2 cells.
}

\begin{abstract}
Vanillin and its analogs have been exploited for their various health benefits. This work aimed to investigate the antioxidant properties and regulatory effects of vanillin rich fraction (VRF) extracted from vanilla pods using the supercritical fluid extraction (SFE) and commercial vanillin on low density lipoprotein receptor (LDLR) and 3-hydroxy-3methylglutaryl-coenzyme A reductase (HMGCR) gene expression in HepG2 cells. The vanillin content in the VRF was $2.6 \%(\mathrm{w} / \mathrm{w})$ obtained at a temperature of $80{ }^{\circ} \mathrm{C}$ and a pressure of 600 bar. The VRF exhibited better antioxidant activity compared to the vanillin in DPPH and BCB tests. LDLR mRNA level was increased significantly by 2, 3 and 1.3 fold in the VRF treated cells at 100, 200 and vanillin treated cells at 100, respectively, compared with untreated cells. On the other hand, the HMGCR mRNA level was decreased significantly by 14, 58 and 13\% respectively, in the VRF treated cells at 100, 200 and V treated cells at 100, respectively, compared with untreated cells. The VRF showed potential antioxidant activity and regulated genes involved in cholesterol metabolism including LDLR and HMGCR in dose-dependent manner.
\end{abstract}

Keyword: Vanillin rich fraction; LDLR; HMGCR; SFE 